\title{
A Divisào de Celulose e Papel do INPA
}

\author{
Antônio A. Corrêa (") \\ Cleusa Maria Corrêa (")
}

\begin{abstract}
Resumo
A Divisão de Celulose e Papel do Instituto Nacio. nal de Pesquisas da Amazônia (INPA) surgiu de um projeto inicial ligado ao Centro de Pesquisas Florestais, idealizado e planejado pela Superintendência do Plano de Valorização Econômica da Amazônia (SPVEA), quando elaborou o seu primeiro Programa para a região Ama. zônica, confiando ao INPA a execução do Projeto. Do projeto inicial até a realidade atual, muitas foram as pessoas e entidades envolvidas e interessadas que contribuíram para a sua concretização. O objetivo da Divisão é fornecer informações técnicas e científicas ao pú. blico das possibilidades das madeiras e materiais $\mathrm{fi}$. brosos da Amazônia, para a produção de celulose e papel e servir de treinamento e orientação para estudantes da região. Já teve a oportunidade de ter dois trabalhos premiados pela Associação Brasileira Técnica de Celulose e Papel. Possui infra-estrutura, instrumentação e recursos humanos para a execução de suas pesquisas e alarga a sua atuação para o campo da xiloquímica.
\end{abstract}

\section{INTRODUÇÃO}

A Divisão de Celulose e Papel do INPA foi criada como parte integrante do Centro de Pesquisas Florestais, idealizado e planejado pela SPVEA (Superintendência do Plano de Valorização Econômica da Amazônia, atualmente Superintendência do Desenvolvimento Econômico da Amazônia, SUDAM), através da Comissão de Planejamento, quando da elabora. ção do Programa de Emergência para 1954. que antecedeu ao primeiro Plano Qüinquenal. No referido Programa, no Relatório da Subcomissão de Recursos Naturais, figurava entre os estudos prioritários um Projeto Celulose (SPVEA, 1954).

A SPVEA confiou então ao Instituto Nacional de Pesquisas da Amazônia a montagem e funcionamento do Centro de Pesquisas Florestais e por meio de convênios anuais injetava recursos para a sua concretização.
O Centro tinha um amplo objetivo, mas dada a falta de recursos e técnicos especializados, ficou limitado em três Seções: Botânica, Silvicultura e Tecnologia da Madeira (Laboratório de Anatomia e implantação do Labora. tório de Celulose e Papel). (Reis, 1958).

\section{EVOLUÇÃO E DESEMPENHO}

Robert Lechthaler, engenheiro técnico em celulose e papel, pesquisador contratado pelo INPA em 1954, elaborou um programa de trabalho para examinar cientificamente 0 aproveitamento das florestas heterogêneas da Amazônia para a fabricação de celulose e papel.

Em uma Mesa Redonda, realizada em 14 de dezembro de 1954, na sede do INPA, à rua Simão Bolivar, 203, Manaus, com a participação do Dr. Renée Cachot, chefe da Missão Florestal da FAO na Amazônia e técnicos do INPA, entre os quais Robert Lechthaler, foi levantado por este pesquisador o tema da possibilidade do aproveitamento de pastas celulósicas de madeira da Amazônia. Informava, então aquela autoridade que em consulta à FAO (Food and Agricultural Organization) de uma organização de países da América Latina, especulava-se, já na época, a viabilidade técnica de implantação de uma fábrica que seria localizada na península de Yucatan no México ou no Território Federal do Amapá no Brasil. Renée Cachot era de opinião que, inicialmen. te, antes de qualquer medida de caráter industrial e comercial, fosse instalada uma Usina Piloto com apoio financeiro do Governo para estudos, idéia esta acatada e defendida peIo INPA.

(*) - Instituto Nacional de Pesquisas da Amazônia, Manaus. 
R. Lechthaler projetou e supervisionou a compra dos equipamentos essenciais, bem como, enquanto aguardava a chegada dos mes. mos, fez o primeiro estudo para o INPA com madeiras da Amazônia, utilizando os equipamentos da fábrica Duratex em Jundiaí, São Paulo e do Instituto Nacional de Tecnologia do Rio de Janeiro.

Hla A demora da chegada dos equipamentos e com a saída de R. Lechthaler em 1958, ficaram os mesmos encaixotados durante longos 7 anos.

Só em 1965, o Dr. Djalma Batista, então diretor do INPA, se empenhou arduamente na tarefa de dar continuidade ao projeto e conseguiu trazer o Dr. W. Overbeck, técnico aposentado do Instituto de Perquisas Tecnológicas de São Paulo, que no período de abril a dezembro do mesmo ano, montou e colocou em funcionamento os equipamentos. Inaugu rada então jubilosamente a Usina Laboratório, com a presença do Excelentíssimo Senhor Presidente da República, General Humberto de Alencar Castelo Branco, que teve a oportunidade de examinar as primeiras folhas de papel de pasta crua, feitas de imbaúba (Cecropis $\mathrm{sp}$ ). Foi sem dúvida uma grande etapa vencida (Fig. 1).

Entretanto a sua instalação ainda era precária, localizada que estava em uma sala do andar térreo de um edifício alugado de 3 andares, onde funcionava a sede do INPA, na rua Guilherme Moreira, n. ${ }^{\circ} 112 / 116$, no centro urbano comercial de Manaus.

Para preparar tecnicamente pessoas que Iriam dirigir as pesquisas, seguindo a política de Djalma Batista, de dar oportunidade a jo. vens da região para se tornarem pesquisadores, na tentativa de suplantar o problema sempre presente da falta de pessoal especializa. do na região e da não permanência de elemen tos extra-regionais no INPA, o que de certo modo prejudicava a continuidade das pesquisas, foi enviado ao Instituto de Pesquisas Tecnológicas em São Paulo, como bolsista do CNPq o Químico Industrial, formado pela Universidade Federal do Pará, Roberto de Freitas Lobato. Lá estagiou durante 1 ano sob a orien-

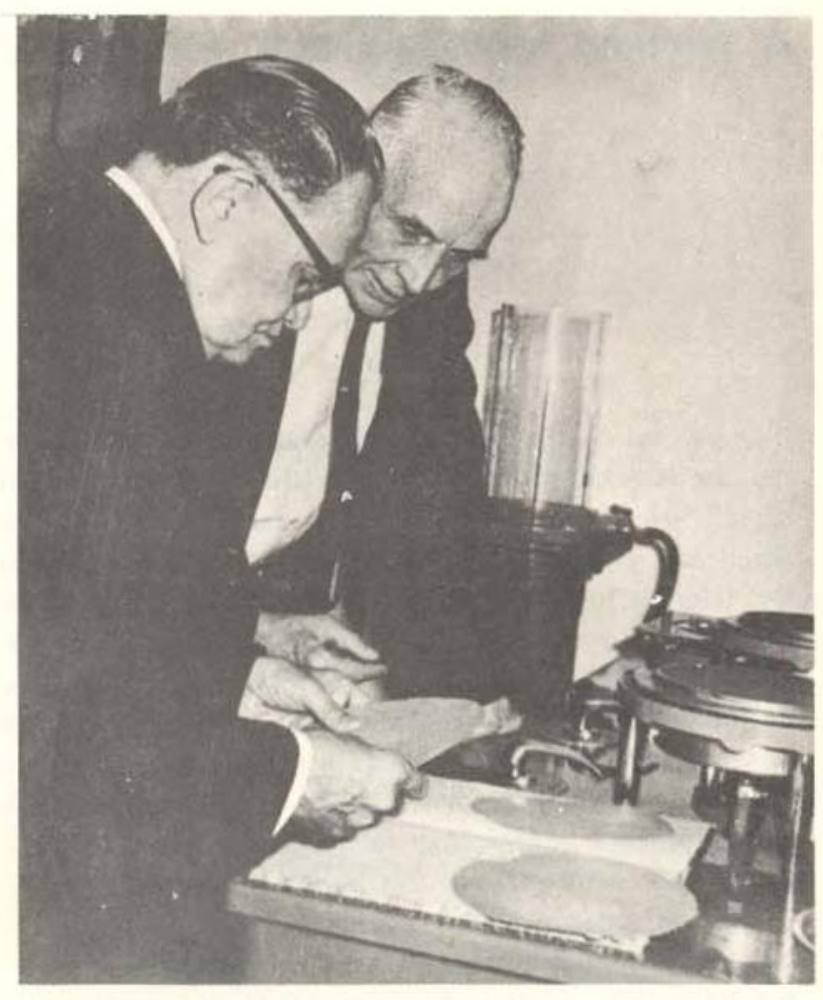

Fig. 1 - Presidente Humberto de Alencar Castelo Branco juntamente com W. Overbeck, examinando as primeiras folhas de papel produzidas no INPA.

tação do Eng. Ovídio da Silva Sallada, chefe da Seção de Celulose e Papel daquele órgão, nessa ocasião. Após esse período de treinamento, foi estabelecido um convênio entre o INPA e o IPT que estipulava a vinda do $\mathrm{Dr}$. Sallada ao INPA (agosto de 67) durante um mês, para orientar as pesquisas iniciais, produzindo-se um trabalho, utilizando-se o "Cardeiro" (Scleronema micranthum) como matériaprima

Posteriormente, vieram agregar-se mais dois químicos da região formados pela Universidade Federal do Pará, Antônio de Azevedo Corrêa, (1968) com estágio no IPT, no Laboratório de Celulose e Papel, como bolsista da SUDAM e Eloy Barbosa Penna Ribeiro (1969), com curso de extensão em Química e Celulo. se e Papel na Universidade de Syracuse, Esta. dos Unidos.

Em 1969, na gestão de Paulo de Almeida Machado, novos e importantes impulsos fo. ram dados ao Projeto, que teve também na 
pessoa do Vice-Presidente do CNPq, Heitor da Silveira Grillo, entusiasta, defensor e admirador do INPA, grandes incentivos.

O ano de 1970 foi marcado com um grande evento profissional, o trabalho Estudos Papeleiros das Madeiras da Amazônia foi laurea do pela Associação Técnica Brasileira de Celulose e Papel, em primeiro lugar, entre os trabalhos apresentados na $\|^{\mathrm{a}}$ Convenção anual. Isto fez com que os autores (Corrêa, Antônio A.; Lobato, Roberto de Freitas e Ribeiro, Eloy Barbosa Penna) fossem agraciados pelo Governador do Estado do Amazonas com a Me. dalha Comemorativa do Tricentenário da Fun. dação da Cidade de Manaus, como agradecimento e incentivo do Amazonas aos pesquisadores (Fig. 2 e 3 ).

Outro grande acontecimento no início da década de 70 , foi a construção da sede pró. pria do INPA, na Estrada do Aleixo, e, conseqüentemente, a base física do atual Laborató. rio e Usina Piloto de Celulose e Papel, cuja área construída total é de $250 \mathrm{~m}^{2}$ de área coberta.

Ainda em agosto de 1970, ocorreu o que se chamou Missão G. Petroff que, através de um programa de cooperação técnica entre o Governo Brasileiro e o Francês, aquele espe. cialista, Chefe da Divisão de Celulose e Papel do Centre Technique Forestier Tropical em Nogentsur-Marne, esteve no INPA, como consultor das pesquisas que estavam sendo desenvolvidas no ramo de celulose e papel.

A presença de G. Petroff (agosto de 1970) no INPA, foi da mais alta relevância, pois, além de sua larga experiência em pesquisa com madeiras tropicais, elaborou um programa de longo prazo para a Divisão, avaliou o plano de trabalho existente, corrigindo distorções, introduzindo técnicas e metodologias mais novas e adequadas às nossas madeiras, orientando a estrutura funcional do Laboratório e ainda sugerindo e facilitando a ida de Antônio A. Corrêa e Eloy Barbosa Penna Ribeiro para estagiarem no Centre Technique Forestier Tropical e no Centre Technique de L'Industrie des papiers, Cartons et Cellulose, durante o período de 12 de setembro de 1971 a janeiro de 1972 como bolsista da ACTIM (Agence pour la Coopération Technique Industrielle et Economique).

O intercâmbio entre o INPA e o CTFT proporcionou a viagem de visita do Diretor do INPA ao CTFT com extensão às suas Estações Experimentais na África e também a Missão Científica que M.R. Catinot, engenheiro flo-

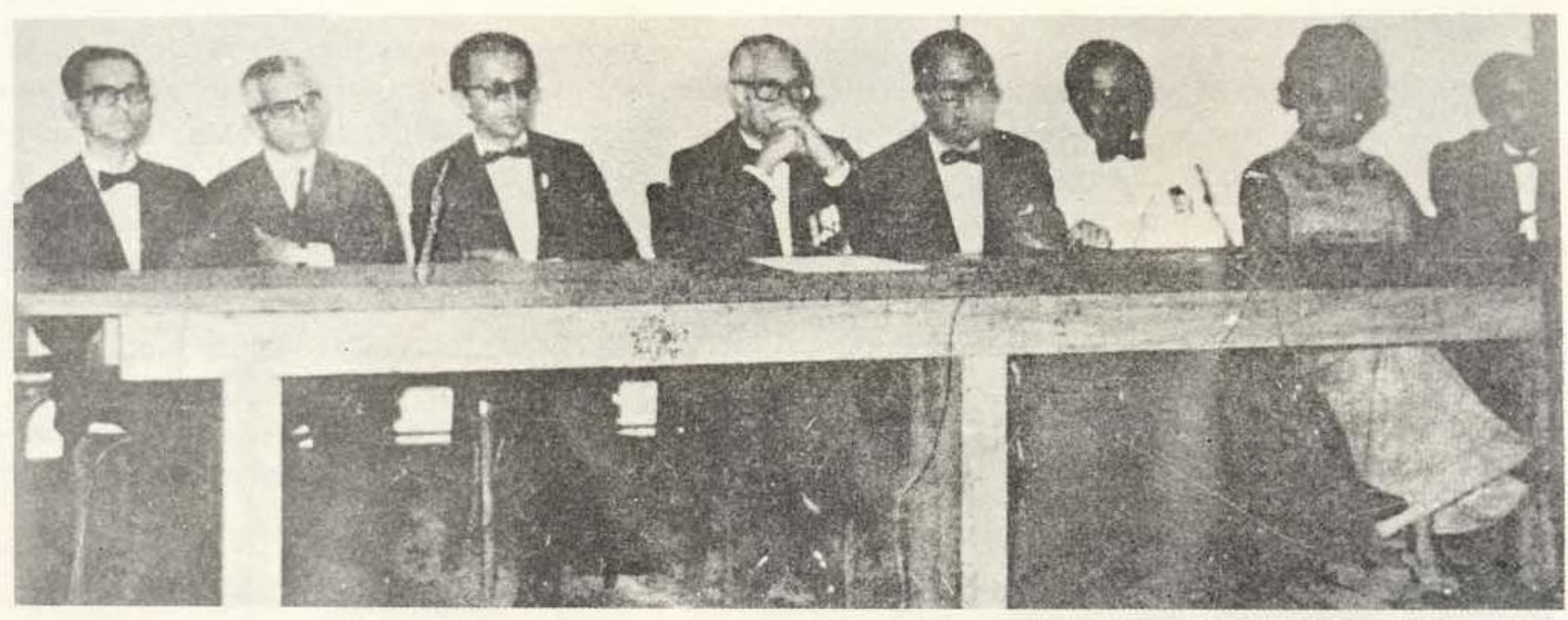

Fig. 2 - Autoridades presentes na solenidade de entrega da medalha Cidade de Manaus aos pesquisadores do INPA, vendo-se da esquerda para direita o Dr. Djaima Batista, Dr. Aderson Dutra, Sr. Elson Farias, Governador Danilo Areosa, Cel. Floriano Pacheco, Dr. Paulo de Almeida Machado, Sra. Cecilia Margarida e o Dr. José Leite Saraiva (Foto publicada na Revista Bom Dia, ano I, número 2, fevereiro de 1971. 


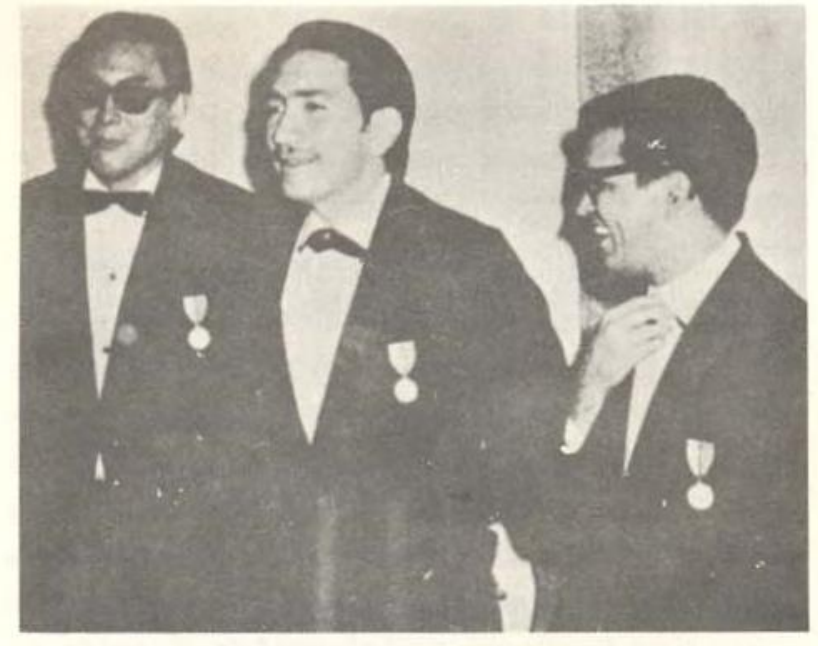

Fig. 3 - Da esquerda para a direita vemos os pesquisadores do INPA Antônio A. Corrêa, Eloy Barbosa Penna Ribeiro e Roberto de Freitas Lobato após serem laureados com a Medalha Comemorativa do Tricentenário da Cidade de Manaus. (Foto publicada na Revista Bom

Dia, Ano I, número 2, fevereiro de 1971.

restal e Diretor do CTFT, realizou em Manaus para orientar as pesquisas florestais efetivadas pelo INPA.

Com a construção da área física do Laboratório e as seguras sugestões de G. Petroff, novos e necessários equipamentos foram sendo adquiridos e hoje podemos orgulhar-nos de possuir um dos laboratórios, nesse ramo, muito bem equipado e estruturado.

Antônio A. Corrêa vem liderando os trabalhos da Divisão desde 1970, cujo escopo básico é fornecer informações técnicas e científicas ao público, das possibilidades das madeiras e materiais fibrosos da Amazônia para a produção de celulose e papel, além de servir de orientação e treinamento para jovens estudantes da região.

O processo de formação e aperfeiçoamento de pessoal continua em andamento. Em 1973, Antônio A. Corrêa, fez Curso de Especialização em Desenvolvimento Regional, Programa Internacional de Formação de Especialistas em Desenvolvimento de Áreas Amazô. nicas, Núcleo de Altos Estudos Amazônicos, Universidade Federal do Pará. Em 1976 fez Mestrado em Tecnologia de Produtos Flores. tais no Curso de Pós-Graduação em Engenharia Florestal do Setor de Ciências Agrárias da Universidade Federal do Paraná, defendendo tese em 1979.

No início de 1975, a Divisão de Celulose teve a oportunidade de participar de um programa de cooperação técnica entre as empresas Kimberly Clark Corporation e APLUB AgroFlorestal da Amazônia S.A., em uma pesquisa que teve como mérito fazer as avaliações das fibras e características das pastas originárias de árvores provenientes de ecossistemas tropicais autóctones, localizado na Amazônia brasileira, na região de Carauari. Naquele evento, o pesquisador Antônio de Azevedo Corrêa, viajou como consultor a convite da empresa americana aos Estados Unidos da América do Norte, em Neenah, Wisconsin, a fim de acompanhar os ensaios e avaliações das pastas.

Por motivos pessoais e conjunturais saíram os outros dois técnicos pioneiros, sendo incorporados mais dois Químicos Industriais formados pela Universidade do Pará, Cláudio Nazareno Reis Luz, aceito como bolsista do CNPq em 1973, depois de fazer o Curso PIATAM, (Programa Intensivo de Adestramen. to para o Trabalho na Amazônia, curso idealizado e executado pelo INPA por iniciativa de Paulo de Almeida Machado em 1970) e Francisco Juvenal Lima Frazão, contratado em 1975

Atualmente, em termos de recursos humanos, a Divisão está estruturada da seguinte forma : 1 Engenheiro Químico (Msc em Tecnologia de Produtos Florestais), 2 Químicos Industriais, cursando mestrado em Tecnologia de Celulose e Papel na Universidade Federal de Viçosa, 1 Economista com curso de Especialização em Desenvolvimento Regional, 6 Auxiliares Técnicos, dispondo assim do instru. mental necessário para a efetivação das pesquisas propostas no seu programa de longo prazo.

\section{PESQUISAS REALIZADAS}

O trabalho que marcou o início das atividades de pesquisa da Divisão, deveu-se a Arens \& Lechthaler (1958), onde aqueles autores descreviam o estudo anatomo-histológico da ma- 


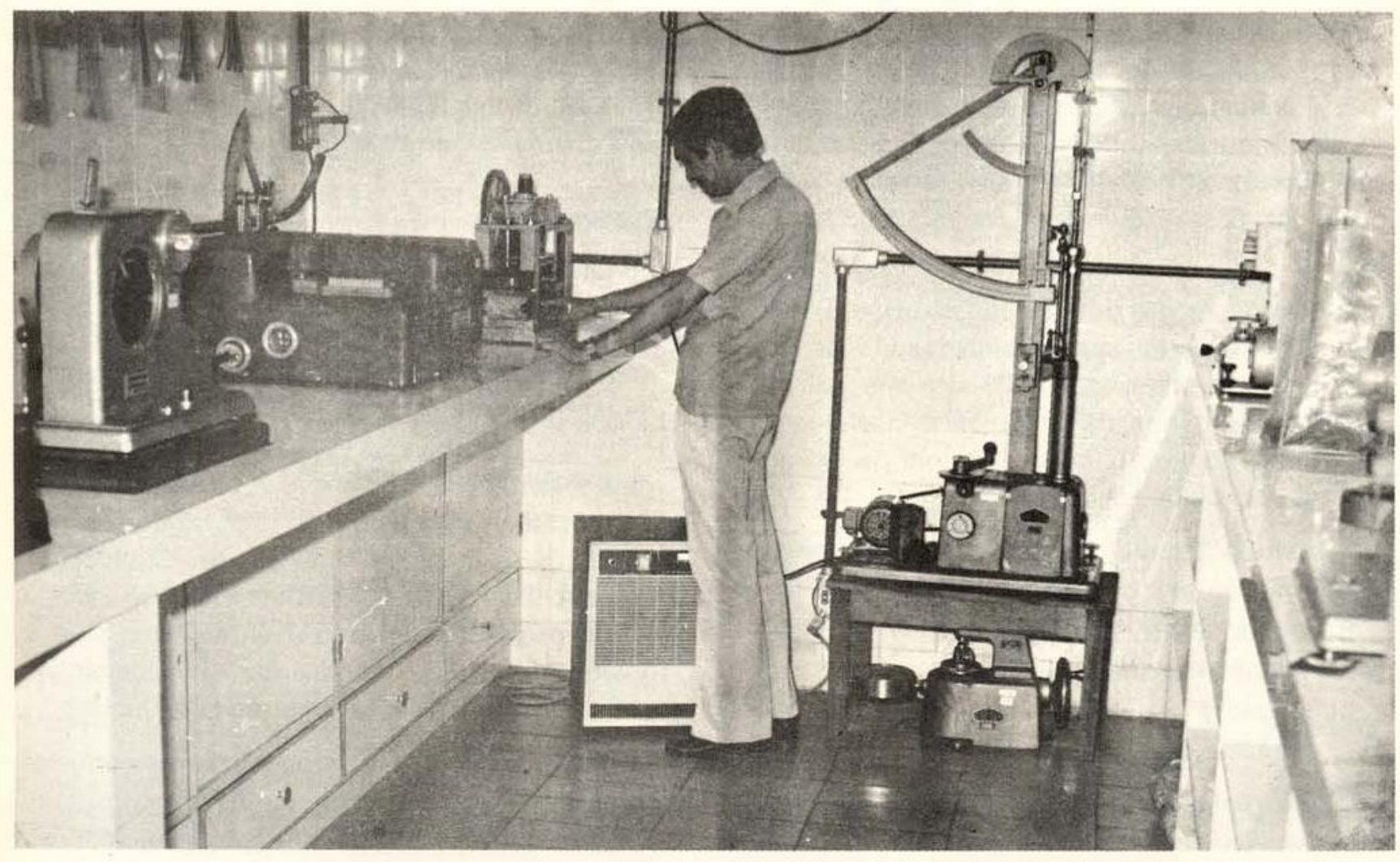

Fig. 4 - Laboratório de testes físico-mecânicos com os equipamentos destinados a testar a resistência do papel.

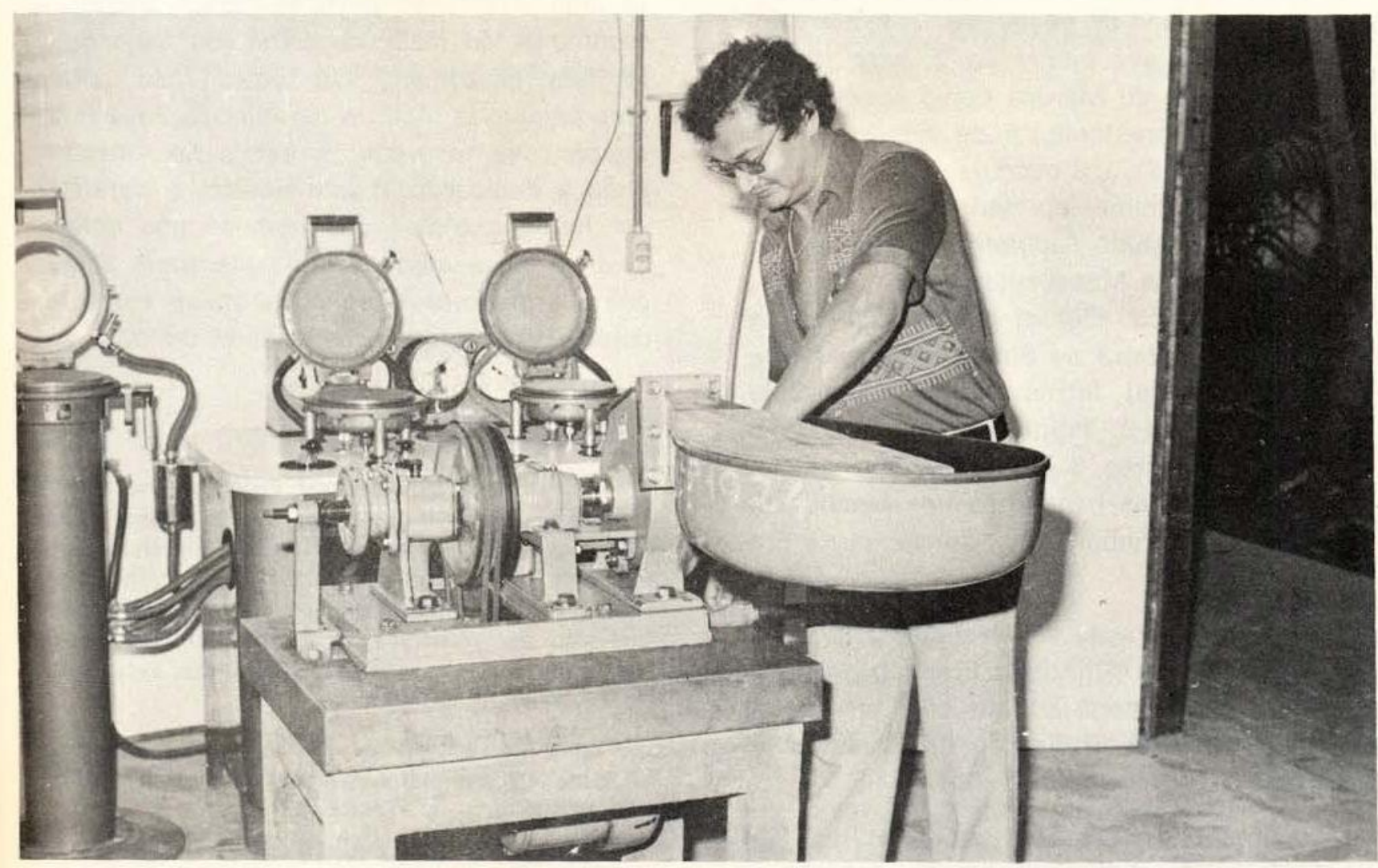

Fig. 5 - Instrumentos utilizados na pesquisa. Moinho Holandesa e formador Rapid Knöoten. 
deira açacu, visando o seu aproveitamento pa. ra a fabricação de celulose. Causas estruturais e conjunturais fizeram que o trabalho sub. seqüente surgisse 12 anos depois. Seus autores (Corrêa et tal., 1970) analizavam a qualidade das pastas químicas de 12 madeiras da estrada Manaus-Itacoatiara.

Com o advento da Missão Petroff, em 1970, a Divisão teve as suas atividades de pesquisas mais sistematizadas, uma vez que, foi elaborado o Programa de Pesquisa de Celulose e Papel para a Amazônia, que dirigia as pesquisas para três linhas distintas:

a) Estudo Papeleiro de Maciços Florestais Amazônicos;

b) Estudo Papeleiro de Essências de Reflo. restamento, Nativas e Exóticas;

c) Estudo Papeleiro de Matérias-Primas Diversas.

Vale realçar que nos dias atuais, estas diretrizes são ainda observadas.

Considerando esta sistematização, a Divisão vem investigando, relatando e divulgando os seus trabalhos de pesquisas, em intervalos com promédio seqüencial de 2 anos. Assim foram o estudo do Marupá como Essência Papeleira de Reflorestamento, de autoria de Corrêa \& Ribeiro (1972), a pesquisa sobre a Trema micrantha (L) Blume efetuada por Ribeiro \& Luz (1973), o Estudo Papeleiro do Maciço Florestal da Estrada Manaus-Itacoatiara, levado a efeito por Corrêa, Ribeiro e Luz (1974), a investigação papeleira do Pinus caribeae (variedade hondurensis) introduzido na Amazônia, destacado com o $3 .^{\circ}$ Prêmio da ABCP em 1975, relatado por Corrêa \& Luz (1976) e a análise das características papeleiras dos bambus do Estado do Acre, redigido por Corrêa, Luz e Frazão (1977).

Como colaboração a estratégia para a Política Florestal na Amazônia Brasileira, Corrêa \& Corrêa (1979) contribuíram com um tópico intitulado Floresta, Utilização de Produtos da Madeira.

Finalmente, a Divisão detém a tese de mestrado, defendida por Corrêa (1979), na Universidade Federal do Paraná, sobre a De- manda por Celulose e Papel e Estudo Compa. rativo de Pastas Celulósicas de Madeiras da Amazônia com Eucalyptus alba Reinw ex Blume e Cimelina arborea Roxb.

\section{CONCLUSÃO}

A Divisão de Celulose e Papel, que há 30 anos foi uma idéia de brasileiros e amazônidas picneiros, hoje é uma realidade adulta e deverá prosseguir, seguindo o exemplo da per. sistência, perspicácia e visão dos que iniciaram a arrancada, procurando novos caminhos. Estes estão sem dúvida, no modelo da sua progênie : a Divisão de Celulose e Química do "Centre Technique Forestier Tropical", desta forma parte-se com todo o entusiasmo para a diversificação das atividades, iniciando-se um projeto na área tecnológica da xiloquímica.

A instalação das áreas complementares concernentes à tecnologia da madeira, no Campus do INPA (secagem, preservação, lâminas e compensado, chapas de fibras, etc.) as quais deverão agrupar-se às já existentes, constituirá uma integração perfeita para o estudo da tecnologgia da madeira, digno da pujança da floresta amazônica. Por outro lado, vira-se uma página da história da valorização e investigação dos recursos florestais da Amazônia, onde a dedicação, o entusiasmo, a persistência forem a tônica dos homens que nela se envolveram e cujo exemplo merecem admiração e orgulho, pois constituiram-se em verdadeiros bandeirantes dos nossos tempo.

\section{SUMMARY}

The Division of Pulp and Paper of the National Amazonian Research Institute (INPA) arose from a project iritially linked to the Forest Research Center. visualized and planned by the Superintendent for Planing and Economic Evaluation of Amazonia (SPVEA). This plan was elaborated in his first program for the Amazonian region, confiring to INPA the execution of the proje:ct.

From the instigation of the project until today many per:sons and entities were involved and interested in contributing to its realization.

The objective of the Division is to furnish technical and scientific information to the public on the possibilities of ivood and fibrous materials from amazonia, on 
the production of pulp and paper, and to serve as a center for training and orientation of students within the region. Two scientific works have been given special awards by the Pulp and Paper Technical Association of Brazil. There is presently available an infrastructure, instruments and human resources for the execution of research and widening of attention to the field of the wood chemical feed-stock products.

\section{REFERENNCIAS BIBLIOGRAFFICAS}

ARENS, K.; LECHTHALER, R.

1958 - Estudo Anatomo-Histológico da madeira açacu, visando o seu aproveitamento para a fabricação de celulose. Publicação do INPA. Série Botânica, 6: 1-27.

CORREA, A.A.; LOBATO, R.F.; RIBEIRO, E.B.P.

1970 - Estudo Papeleiro de Madeiras da Amazônia. O Papel (XXX 1) - Dezembro, 95: 134.

CORREA A.A.; RIBEIRO, E.B.P.

1972 - O Marupá como Essência Papeleira de Reflorestamento. Acta Amazonica, 2 (3): 33-91.

CORREA, A.A.; RIBEIRO, E.B.P.; LUZ, C.N.R.

1974 - Estudo Papeleiro de Maciços Florestais Amazônicos 1 - Maciço Florestal da Estrada Manaus-Itacoatiara. Acta Amazonica, 4 (2): 23-46.

CORREAA, A.A.; LUZ, C.N.R

1976 - Essência Papeleira de Reflorestamento II O Pinus caribeae (Variedade Hondurensis) introduzido na Amazônia. Acta Amazonica, 6 (1): $75-98$,

CORREA, A.A.; LUZ, C.N.R.; FRAZÃO, F.J.L.

1977 - Características Papeleiras dos Bambus do Estado do Acre. Acta Amazonica, 7 (4): 529-550.

CORREA, A.A.

1979 - A Demanda por Celulose e Papel e Estudo Comparativo de Pastas Celulósicas de FoIhosas da Amazônia com o Eucalyptus alba Reinw ex Blume e Gmelina arborea Roxb. Tese de Mestrado apresentada no curso de Pós-Graduação em Engenharia Florestál. Setor de Ciências Agrárias da Universidade do Paraná.

CORREA, A.A.; CORREA, C.M.

1979 - Floresta, Utilização de Produtos de Made!ra. Acta Amazonica, Suplemento 9 (4): 155164.

REIS, A.C.F.

1958 - Relatório do INPA ao CNPq. Publicação avulsa $n: 13$ (mimeografado).

RIBEIRO, E.B.P.; LUZ, C.N.R.

1973 - Estudo Papeleiro da Trema micrantha (L) Blume. Acta Amazonica, 3 (3): 45-50.

Superintendência do Plano de Valorização Econômica da Amazônica

1954 - Programa de Emergência. Setor de Coordenação e Divulgação, 5-169. 